\title{
Haemophagocytic Syndrome in a 19-Year-Old Male with Plasmodium falciparum Malaria
}

\author{
Rembert A. Mertens ${ }^{a}$, Sabine D. Allard ${ }^{a}$, Lucie Seyler ${ }^{a}$, Kristin Jochmans $^{b}$, Patrick Lacor ${ }^{a}$ \\ ${ }^{a}$ Department of Internal Medicine and Infectious Diseases, Universitair Ziekenhuis Brussel, Brussels, \\ Belgium \\ ${ }^{\mathrm{b}}$ Laboratory of Hematology, Universitair Ziekenhuis Brussel, Brussels, Belgium
}

\begin{abstract}
:
Objectives: Infectious agents triggering haemophagocytic lymphohistiocytosis (HLH) primarily involve the herpes virus group. We report a case of HLH precipitated by Plasmodium falciparum.

Materials and methods: Clinical and laboratory findings in a patient presenting with fever were collected. After confirmation of acute malaria, anti-malarial treatment was administered.

Results: Despite initial favourable evolution, the patient developed fever again together with a worsening of the haematological parameters and increased ferritin levels. A bone marrow biopsy confirmed the diagnosis of $\mathrm{HLH}$.
\end{abstract}

Conclusion: This case illustrates that HLH should be considered in the differential diagnosis of acute malaria in patients with persisting fever and pancytopenia.

Keywords: Plasmodium falciparum, hemophagocytic lymphohistiocytosis

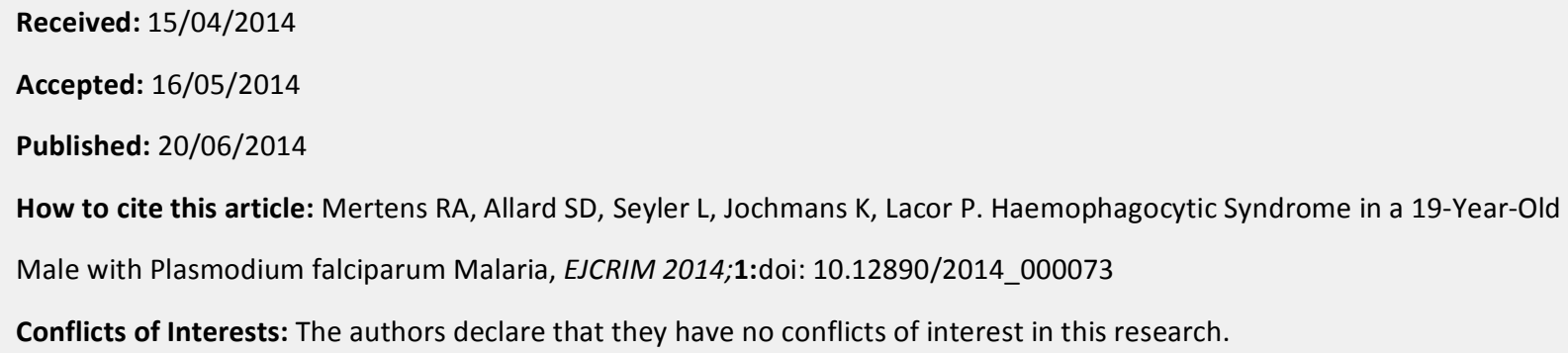




\section{Introduction}

Haemophagocytic lymphohistiocytosis (HLH) is a clinical entity characterized by fever, pancytopenia and haemophagocytosis in bone marrow and possibly other tissues ${ }^{1}$. It can occur as a primary syndrome, referred to as familial $\mathrm{HLH}$, or as a syndrome secondary to an underlying autoimmune, neoplastic or infectious disease, referred to as reactive HLH. Infectious agents triggering reactive HLH primarily involve the herpes virus group, predominantly Epstein-Barr virus (EBV). Here we report a patient presenting with reactive HLH precipitated by Plasmodium falciparum infection.

\section{Case report}

A 19-year-old Belgian male was admitted to hospital because of a fever (up to $40^{\circ} \mathrm{C}$ ), which he had had for 2 days. He complained of headache, diffuse muscular pain and nausea. Four days before the onset of fever, he had returned from the Democratic Republic of Congo, where he had stayed for 3 weeks. He had not taken malaria prophylaxis.

There was nothing of significance in his medical history. Physical examination showed a man who appeared ill, with a tympanic temperature of $39^{\circ} \mathrm{C}$. His blood pressure was $135 / 74 \mathrm{mmHg}$ and rate was 114 beats/min. The cervical lymph nodes were slightly swollen. The rest of the physical exam was normal.

Laboratory tests showed elevated levels of C-reactive protein and cytolytic liver enzymes as well as thrombocytopenia (Table 1). Chest X-ray was normal.

The clinical suspicion of acute malaria was confirmed by a thick blood smear, showing the presence of $P$. falciparum with only rare erythrocytes parasitized. A supportive treatment and oral quinine sulphate (500 $\mathrm{mg}$ tid), to be followed by doxycyclin from day 3 onwards, was initiated. Because of gastrointestinal intolerance, this schedule was changed to atovaquone $250 \mathrm{mg} /$ proguanil $100 \mathrm{mg}, 4$ tablets daily for 3 consecutive days, starting on day 3. Abdominal ultrasound, performed on day 2 of admission, showed slight splenomegaly $(12.7 \mathrm{~cm})$.

Although the clinical evolution was initially favourable, from day 3 onwards, the patient started having a high fever again, up to $39^{\circ} \mathrm{C}$. He complained of headache and there was intermittent confusion with signs of meningismus. Gradual worsening of the haematological parameters was observed with deepening neutropenia and an increasing percentage of atypical lymphocytes (Table 1). 


\begin{tabular}{|c|c|c|c|c|c|c|c|c|c|}
\hline & & \multicolumn{8}{|l|}{ Day } \\
\hline & & 0 & 1 & 2 & 3 & 4 & 5 & 6 & 8 \\
\hline \multicolumn{2}{|c|}{ Maximum body temperature $\left({ }^{\circ} \mathrm{C}\right)$} & 39.0 & 40.0 & 38.2 & 39.0 & 37.5 & 37.5 & 36.4 & 36.5 \\
\hline & Normal Values & & & & & & & & \\
\hline Haemoglobin (g/dl) & $13.0-16.5$ & 15.2 & 14.0 & 12.8 & 12.3 & 11.9 & 11.4 & 10.8 & 10.8 \\
\hline Platelets $\left(\times 10^{3} / \mathrm{mm}^{3}\right)$ & $158-450$ & 79 & 52 & 30 & 27 & 43 & 50 & 84 & 194 \\
\hline $\begin{array}{l}\text { White blood cells } \\
\left(\times 10^{3} / \mathrm{mm}^{3}\right)\end{array}$ & $3.6-9.6$ & 3.5 & 3.7 & 1.5 & 1.8 & 3.8 & 3.9 & 4.5 & 6.7 \\
\hline Neutrophils $\left(\times 10^{3} / \mathrm{mm}^{3}\right)$ & $1.4-6.7$ & 2.9 & 3.1 & 0.8 & 0.7 & 1.1 & 1.3 & 1.7 & 2.6 \\
\hline Atypical lymphocytes (\%) & 0.0 & 0.0 & 0.0 & 14.5 & 21.0 & 12.0 & 7.5 & 5.0 & 4.0 \\
\hline Creatine kinase (U/I) & $<145$ & 288 & nd & 6604 & 5602 & 2250 & 813 & 466 & 205 \\
\hline LDH (U/I) & $241-549$ & 934 & nd & 3719 & 1934 & 1196 & 862 & 859 & 809 \\
\hline $\operatorname{ALT}(U / I)$ & $21-72$ & 111 & nd & 792 & 500 & 409 & 244 & 150 & 65 \\
\hline AST (U/I) & $16-48$ & 100 & nd & 592 & 430 & 366 & 288 & 235 & 146 \\
\hline Y-GT (U/I) & $14-47$ & nd & nd & 64 & 76 & 83 & 88 & 98 & nd \\
\hline$A F(U / I)$ & $65-220$ & nd & nd & 110 & nd & 148 & 176 & 233 & 158 \\
\hline Total bilirubin (mg/dl) & $0.2-1.0$ & nd & nd & 4.94 & 4.83 & 3.93 & 2.67 & 2.10 & 1.59 \\
\hline Direct bilirubin (mg/dl) & $<0.5$ & nd & nd & 3 & 3.2 & 2.3 & 1.6 & 1.1 & 0.6 \\
\hline Fibrinogen (mg/dl) & $180-400$ & nd & nd & 375 & 40 & & & 291 & \\
\hline CRP (mg/l) & $<5$ & 94.1 & 131.0 & 236.0 & 172.0 & 89.4 & 52.7 & 33.8 & 14.7 \\
\hline PT (\%) & $>70$ & 62 & nd & 68 & 76 & 87 & 94 & 91 & nd \\
\hline Ferritin $(\mu \mathrm{g} / \mathrm{I})$ & $30-400$ & nd & nd & nd & nd & 4102 & nd & nd & nd \\
\hline Fasting triglycerides (mg/dl) & $<150$ & nd & nd & 467 & 727 & nd & nd & nd & nd \\
\hline
\end{tabular}

LDH, lactate dehydrogenase; AST, aspartate aminotransferase; ALT, alanine aminotransferase; $\gamma$-GT, gamma-glutamyltransferase; AF, Alkaline posphatase or ALP; CRP, C-reactive protein; PT, prothrombin time; nd, not done

Table 1: Evolution of tympanic temperature and laboratory parameters

New blood cultures remained negative. A thick blood smear showed persistently low falciparum parasitaemia. Serologic studies were compatible with passed infection with EBV and cytomegalovirus.

This clinical picture caused the suspicion of $\mathrm{HLH}$. Triglycerides and ferritin levels turned out to be strongly increased (Table 1). A bone marrow biopsy, performed on day 4, was normocellular with 
megakaryocytic hyperplasia and a markedly increased number of benign-appearing histiocytes without nuclear atypia and exhibiting striking haemophagocytosis (Fig. 1).
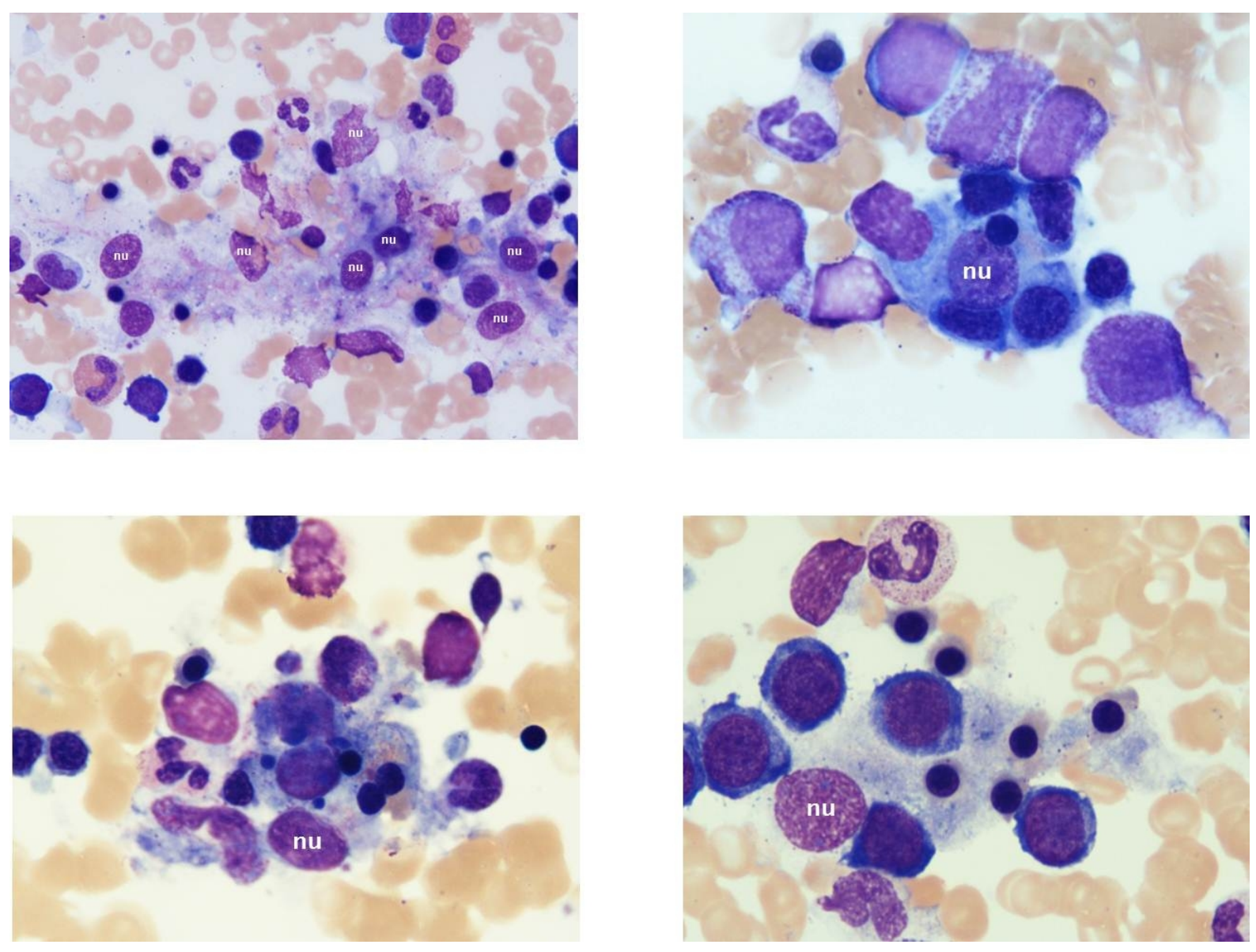

Figure 1: Bone marrow biopsy

Bone marrow aspirate smears (May-Grünwald-Giemsa staining) demonstrate marked haemophagocytosis. Benign-appearing histiocytes contain small, round nuclei (nu) with condensed chromatin. The cytoplasm is abundant with ingestion of mature and immature haematopoietic elements, mainly erythroblasts.

According to the initial clinical evolution and the findings on blood smear, no other active treatment was considered. Broad-spectrum antibiotics (a combination of cefepime and amikacine intravenously) were given as empirical coverage during febrile neutropenia.

From day 4 onwards, fever resolved and the haematological parameters began to improve (Table 1). 
Antibiotherapy was stopped on day 6 and on day 8 the patient left the hospital in a good, asymptomatic condition.

\section{Discussion}

The term haemophagocytosis, first reported in 1939, describes the pathologic finding of activated monocytes, macrophages and histiocytes engulfing erythrocytes, leukocytes and platelets in bone marrow and other tissues ${ }^{1,2}$.

Familial HLH is inherited in an autosomal recessive manner with symptoms usually evident within the first years of life $^{3}$. The association between HLH and infectious diseases can be challenging for the clinician because both reactive and familial HLH are often precipitated by infections. Moreover, HLH may mimic infectious illnesses, such as overwhelming bacterial sepsis, or it may obscure the diagnosis of a precipitating, treatable infectious illness. Our patient developed signs of HLH within 2-3 days after the diagnosis of acute malaria. No other possible triggering infection could be demonstrated. HLH was suspected on clinical grounds, with a differential diagnosis of acute malaria evolving into "severe malaria" or malaria complicated by bacterial septicaemia.

Although the underlying pathophysiologic mechanisms remain unclear, it is thought that all HLHs are due to some form of functional impairment of cytotoxic T lymphocytes and natural killer cells, resulting in the inability to clear the antigenic stimulus and to turn off the inflammatory response, ultimately leading to hypercytokinaemia characteristic of $\mathrm{HLH}^{4}$. However, the exact mechanisms by which abnormal cytokine elaboration results in HLH are still elusive. Once the cytokine cascade has been triggered, HPS may continue to proceed independently of the presence of an initial trigger.

The diagnostic criteria for HLH include clinical, laboratory and histopathologic features. ${ }^{5}$. Fever and splenomegaly are the most common clinical signs but hepatomegaly, lymphadenopathy, jaundice and rash have also been described. Encephalopathy, meningismus and seizures are the most commonly reported central nervous manifestations. The most prominent laboratory abnormality noted is cytopenia. Blood chemistry may suggest haemolysis and most patients have hypertriglyceridaemia as well as marked elevation of serum ferritin. Serum fibrinogen is typically low. Histopathologically, haemophagocytosis can be demonstrated in bone marrow, spleen, liver and lymph nodes and occasionally in the central nervous system and the skin. In our patient, HLH was suspected because of cytopenia affecting two lineages as well as prominently elevated serum ferritin and triglyceride levels. A bone marrow smear allowed confirmation of the diagnosis.

For patients with reactive HLH associated with pathogens other than EBV, supportive care and treatment of the underlying infection is associated with recovery in $60 \%-70 \%$, as was the case in our 
patient ${ }^{5}$. EBV-associated HLH is almost universally fatal if untreated. Therefore, patients should be treated with combination chemotherapy and immunotherapy ( $\mathrm{HLH}-94$ treatment protocol), regardless of whether they are thought to have familial HLH. However, the distinction between reactive and familial HLH triggered by a viral infection is important as allogeneic haematopoietic stem cell transplantation is the only curative therapy in patients with familial HLH who attain remission.

This case illustrates that HLH should be considered in the differential diagnosis of acute malaria patients with persisting fever and pancytopenia. Early recognition of this rare complication enables the clinician to consider timely immunosuppressive treatment in case there is no spontaneous recovery and successful treatment of the underlying malaria attack alone.

\section{Learning Points}

- HLH may mimic infectious illnesses.

- HLH should be considered in the differential diagnosis of acute malaria in patients with persisting fever and pancytopenia.

\section{References}

1. Janka GE. Familial and acquired hemophagocytic lymphohistiocytosis, Annu Rev Med 2012;63:233-246.

2. Scott R, Robb-Smith A. Histiocytic medullary reticulosis, Lancet 1939;2:194-198.

3. Zhang K, Filipovich AH, Johnson J, Marsh RA, Villanueva J. Hemophagocytic lymphohistiocytosis, familial, in Pagon RA, Adam MP, Bird TD, Dolan CR, Fong CT, Stephens K (eds) GeneReviews. Seattle, WA: University of Washington, 1993.

4. Rosado FG, Kim AS. Hemophagocytic lymphohistiocytosis: an update on diagnosis and pathogenesis, Am. J. Clin. Pathol. 2013;139:713-727.

5. Henter JI, Horne A, Arico M, Egeler RM, Filipovich AH, Imashuku S et al. HLH-2004: diagnostic and therapeutic guidelines for hemophagocytic lymphohistiocytosis, Pediatr Blood Cancer 2007;48:124-131. 